\title{
Estimate of sludge floc permeability
}

\author{
R.M. Wu, G.W. Tsou, D.J. Lee* \\ Department of Chemical Engineering, National Taiwan University, Taipei 106017, Taiwan
}

\begin{abstract}
This paper provides an estimate of the waste-activated sludge floc interior permeability on the basis of observing the motion of individual floc moving vertically towards an impermeable flat plate. The fluid flow fields surrounding and inside the floc were modeled numerically, from which the hydrodynamic drag force was calculated. The experimental data correlate with the numerical solutions regardless of the floc Reynolds number. Over the floc size range investigated in this paper, the permeability is noted to be approximately proportional to the square of floc size, ranging from $2.5 \times 10^{-9}$ to $9 \times 10^{-6} \mathrm{~m}^{2}$ for floc size ranging from 150 to $10000 \mu \mathrm{m}$. The structure of waste-activated sludge floc is proposed as a multi-fractal. Permeability measured in this work is thereby mainly attributed to the global structure level of the flocs. (C) 2000 Elsevier Science B.V. All rights reserved.
\end{abstract}

Keywords: Sludge floc; Permeability; Structure; Multi-fractal

\section{Introduction}

Floc size and density are two of the factors that most influence the performance of floc processes. A free-settling test has been widely employed to estimate these two factors [1-8]. Based on the so-constructed floc size/density relationship, a floc has been proposed as a highly porous fractal-like aggregate made of many primary particles [4-6,9]. The aggregates generated in water and wastewater treatment processes would exhibit a fractal dimension ranging between 1.4 and 2.8 [4]. Physical and chemical treatment may affect the floc structure, and therefore the corresponding density and the fractal dimension $[10,11]$.

Fig. 1 illustrates a microscopic photograph of a wasteactivated sludge floc approximately $50 \mu \mathrm{m}$ in size. In Fig. 1a, the appearance of the floc is loosely structured and highly irregular in shape. In Fig. 1b, the same floc is ink-stained, with the white portion representing the water-proof regime. Comparing Fig. 1a,b reveals that a great portion of the floc's interior is void, which allows easy moisture permeation [12].

The knowledge regarding the hydrodynamic drag force exerted on a floc is necessary for predicting its motion. The drag force exerted on a floc depends on several unknowns, e.g. drag coefficient [7], primary particle size [10], and the correction factor for advection flow [13,14]. Lee et al. [15] provided a discussion of this issue. To evaluate the correction factor, the floc permeability $k$ must be known a priori. However, since the floc is very fragile and tends to break

\footnotetext{
${ }^{*}$ Corresponding author. Tel.: +886-2-23625230; fax: +886-2-23623040. E-mail address: djlee@ccms.ntu.edu.tw (D.J. Lee).
}

down under shear, the floc interior permeability cannot be accurately evaluated by the conventional column test (which is made in a filled column with flow-through fluid).

In the literature, empirical models are usually used to evaluate the floc permeability. For example, Matsumoto et al. [16] employed the Davies correlation for estimating floc permeability. Li and Ganczarczyk [6] compared the permeabilities calculated from the Carman-Kozeny equation and Davies correlation. Huang [7] employed the Brinkman model for permeability estimation. Six of the widely employed permeability models are summarized in Lee et al. [15], Brinkman, Carman-Kozeny, Happel (sphere), Happel (fibrous), Davies and Iberall, which are all of the same form: $k=f(\varepsilon) \times d_{\mathrm{p}}^{2}$, where $k$ is the permeability of floc, $\varepsilon$ is the floc porosity, and $d_{\mathrm{p}}$, the diameter of primary particles that constitute the floc. As addressed in Lee et al. [15], these two quantities are not available for a real sludge process and are usually taken as fitting parameters. Rogak and Flagan [17] also proposed an expression for permeability based on the fractal nature of a floc. Chellam and Wiesner [18] examined Rogak and Flagan's model, and noted that an additional factor, the fractal dimension, markedly affects the permeability. For a specific floc, the $k$ values calculated based on different models can vary by two to three orders of magnitude.

Wu et al. [19] adopted a hydrodynamic approach to estimate the floc interior's permeability. The underlying idea is simple: a porous floc subjected to a (mild) change in hydrodynamic environment should behave differently to a nonporous object of the same size and overall weight. By comparing their responses, the information for the floc interior permeability can be estimated. The next section briefly 


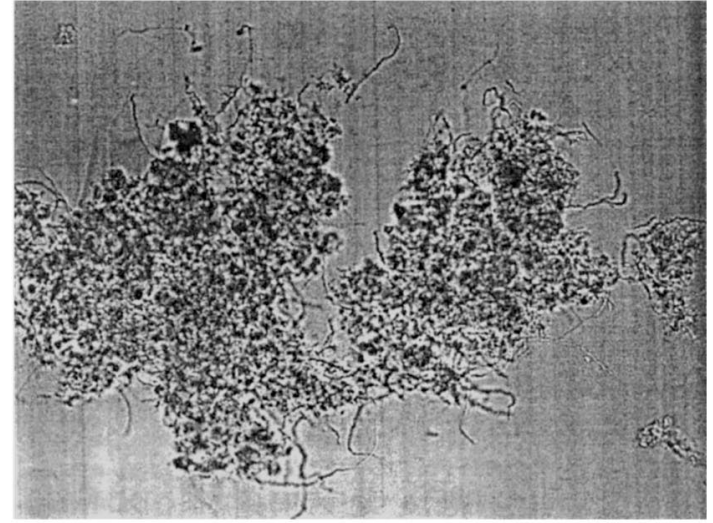

(a)

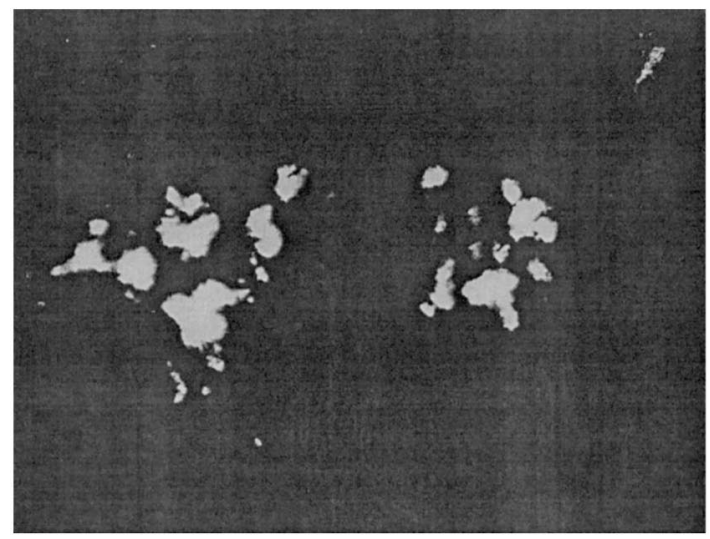

(b)

Fig. 1. Microscopic photographs of activated sludge floc $(100 \times)$. (a) Top, appearance of floc; (b) bottom, ink-stained photograph.

illustrates the basic idea with a floc moving toward a flat plate as an illustrative example.

\section{Sphere moving toward a flat plate}

We herein consider objects of spherical shape only.

A nonporous sphere of diameter $d$ moving very slowly at a velocity $V_{\mathrm{t}}$ through an infinite medium of viscosity $\mu$ would experience a hydrodynamic resistance of $F_{\mathrm{s}}$ (Stokes' law, $\left.=3 \pi d \mu V_{\mathrm{t}}\right)$. When the sphere is moving toward an impermeable flat plate, the hydrodynamic drag $\left(F_{\mathrm{p}}\right)$ would increase $\left(F_{\mathrm{p}}>F_{\mathrm{s}}\right)$ [20]. $F_{\mathrm{p}}$ increases beyond bound as the gap between the floc and plate tends to zero.

For a porous sphere, its drag force to move in an infinite medium $\left(F_{\mathrm{f}, \mathrm{s}}\right)$ would be less than that for a nonporous sphere $\left(F_{\mathrm{s}}\right)$. The ratio $F_{\mathrm{f}, \mathrm{s}} / F_{\mathrm{s}}$ is the correction factor $\Omega$. For a highly porous sphere moving very slowly in a quiescent medium, Neale et al. [21] derived the analytical expression for correction factor $\Omega$ based on the Brinkman model:

$\Omega=\frac{2 \beta^{2}[1-\tan h(\beta) / \beta]}{2 \beta^{2}+3[1-\tan h(\beta) / \beta]}$ and $\beta=d / 2 \sqrt{k}$. Matsumoto and Suganuma [22] experimentally verified Eq. (1).

When a porous sphere is moving towards an impermeable flat plate instead, the fluid in the gap can flow through the sphere's interior. The creeping flow field $(R e \ll 1)$ and the associated drag force $\left(F_{\mathrm{f}, \mathrm{p}}\right)$ have been given analytically by Payatakes and Dassios [23], and corrected by Burganos et al. [24], in which Darcy's law was assumed for the floc interior fluid field. They noted that the correction factor $\Omega$ approaches its corresponding plateau value $(<1)$ under a free-settling condition as the gap between the object and the flat plate $(h / d)$ becomes large $(>10)$. As the gap decreases, the factor $\Omega$ increases. However, it does not go to infinity as the gap tends to zero, but remains finite. This is the reason why particle clusters are easier to trap by a collector than are dense particles [25]. At the extreme condition of zero $\beta$, the drag force would not increase at all when the sphere gradually touches the plate ('no floc' limit).

Consider a sphere that is released from the far top end and moving freely towards (downwards) a bottom flat plate. If the floc Reynolds number is not very large $(<100)$, the transient effect can be safely neglected and the process can be assumed pseudo-steady state. Under such a circumstance, the sum of gravity force, buoyancy force and the drag force is zero, i.e. at any instant along the floc's travel, the following equality holds:

$F_{\text {gravity }}-F_{\text {buoyancy }}=F_{\text {drag }}=\Omega F_{\mathrm{s}}$

The gravity and the buoyancy forces are kept unchanged for a given sphere. As a result, the product $\Omega F_{\mathrm{s}}$ would be constant along the sphere's travel towards the plate. Since $\Omega$ increases with decreasing gap, the sphere has to decrease down its speed to reduce the corresponding $F_{\mathrm{S}}$ (a function of $\left.V_{\mathrm{t}}\right)$. When the sphere is far away from the plate, the terminal velocity is $V_{0}$. The corresponding drag force is $\Omega_{0} F_{\mathrm{s} 0 \text {. As }}$ the floc is closer to the plate where the moving speed has reduced to $V$, the drag force becomes $\Omega F_{\mathrm{s}}$. Consequently, after consulting Eq. (2), we have:

$$
\frac{V}{V_{0}}=\frac{\Omega_{0}}{\Omega}
$$

Estimating $V$ along its travel towards the flat plate can give a measure of the ratio $\Omega_{0} / \Omega$ on the basis of Eq. (3). The floc permeability can be estimated by comparing the $\Omega_{0} / \Omega$ versus $h / d$ relationship if it were available.

The solutions of Payatakes and Dassios [23], and Burganos et al. [24] are strictly valid for a slowly moving sphere with an interior porosity less than, say, 0.5 (with which the Darcy law can be applied) [26]. However, in many applications involving sludge flocs, the Reynolds number is not always much less than unity. In contrast, as Lee et al. [15] reveal, in treating waste-activated sludge flocs of a diameter greater than, say, $500 \mu \mathrm{m}$, the floc's Reynolds number can be several tens. This value can be even greater when considering other sludge flocs, such as polymer flocculated clay sludge flocs. Furthermore, the floc interior is found 
highly porous via mass balance calculations of a porosity up to $99 \%$ [15], which is much beyond the applicable range of Darcy law. In the next section, the numerical scheme to obtain the $\Omega_{0} / \Omega$ versus $h / d$ relationship is described.

\section{Numerical solutions}

Fig. 2 depicts schematically the flow process [19]. We herein assume the sphere to have an interior permeability of $k\left(\mathrm{~m}^{2}\right)$. The governing equation for the fluid velocity $\vec{u}_{\mathrm{p}}(\mathrm{m} / \mathrm{s})$ within the porous sphere, taking into account the viscous effect, is the so-called combined Darcy-Brinkman law, stated as:

$\frac{\mu}{k} \vec{u}_{\mathrm{p}}+\nabla p=\mu \nabla^{2} \vec{u}_{\mathrm{p}}$

For the surrounding Newtonian fluid field, the governing equations are the steady-state Navier-Stokes equations, which can be stated as follows:

$\rho\left(\vec{u}_{\mathrm{f}} \cdot \nabla\right) \vec{u}_{\mathrm{f}}+\nabla p=\mu \nabla^{2} \vec{u}_{\mathrm{f}}$

where $\vec{u}_{\mathrm{f}}(\mathrm{m} / \mathrm{s})$ is the fluid velocity. Notably, the incorporation of the inertial term in Eq. (5) leads to nonlinear differential equations that require numerical solution. Wu and Lee [27] have discussed the domain to solution and the boundary conditions.

The general-purpose computational fluid dynamics program FIDAP 7.5 (FDI Inc., USA) solved the governing equations (Eqs. (4) and (5)), together with the associated boundary conditions. The FIDAP software employs a finite element method with bilinear, four-node quadrilateral elements. The maximum relative error was set at $10^{-3}$. Comparisons with the analytical solution reveal that the numerical determined hydrodynamic drag force subsequently evaluated contains a maximum relative error less than $5 \%$.

The calculated streamlines and velocity fields at different $h / d$ ratios are available in $\mathrm{Wu}$ and Lee [27]. Fig. 3 sum-

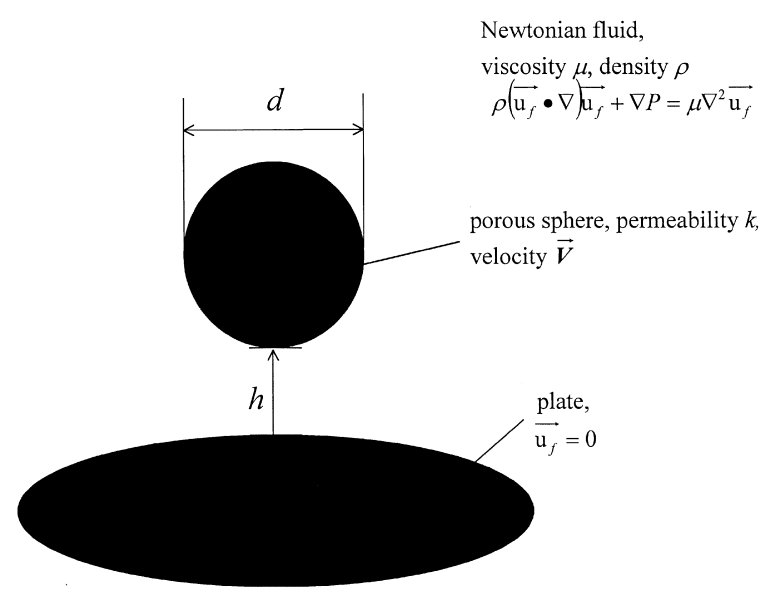

Fig. 2. Schematics of the physical system in computation.
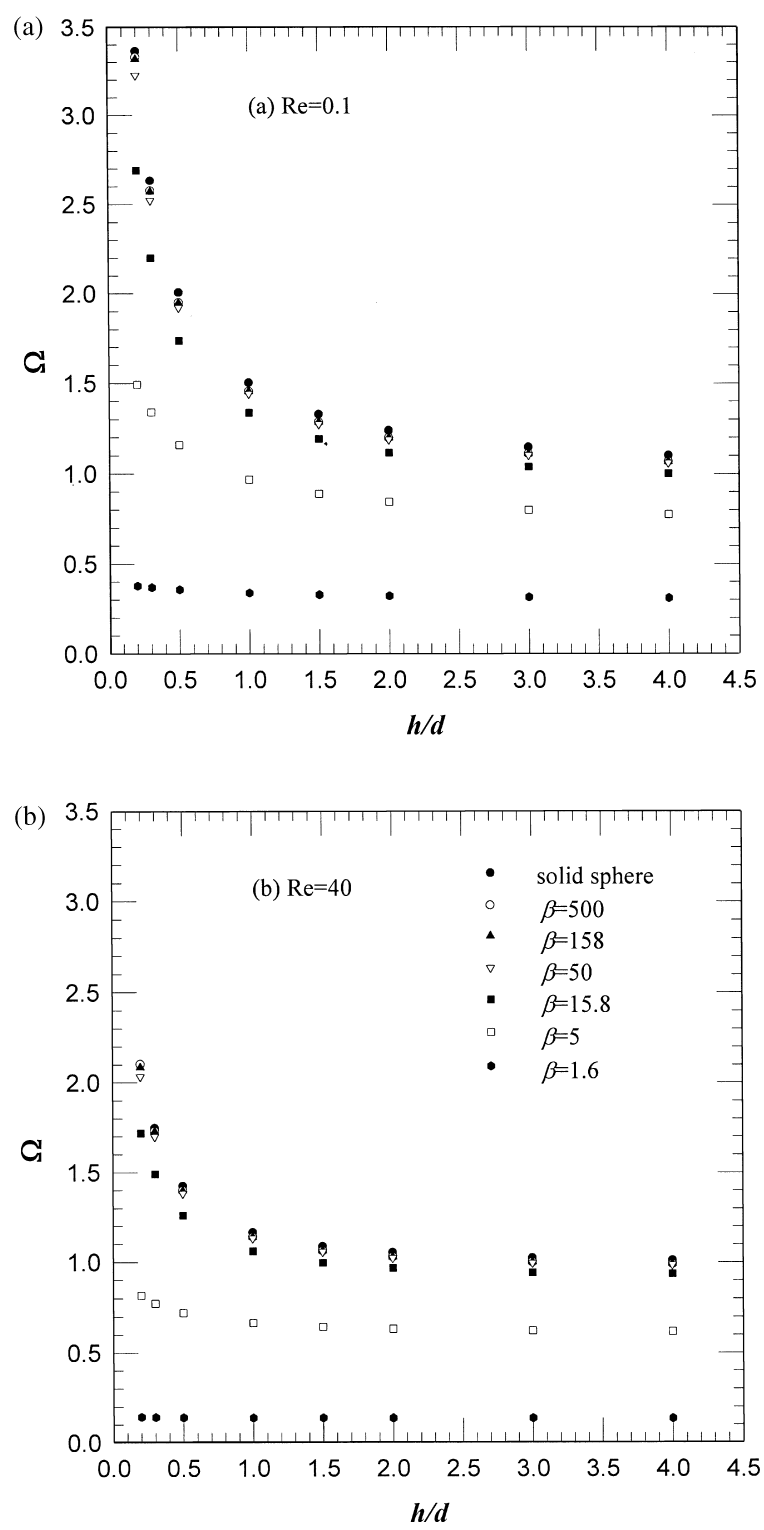

Fig. 3. $\Omega$ versus $h / d$ plot. (a) $R e=0.1$; (b) $R e=40$.

marizes the calculated $\Omega$ versus $h / d$ relationship at various $\beta$ values and $R e=0.1$ or 40 . First note that as $\beta$ exceeds approximately $50, \Omega$ values coincide with that of a nonporous sphere, denoting that the porous sphere can be taken as a nonporous sphere herein. At reduced $\beta$ values $(<20)$, the corresponding $\Omega$ deviates markedly from unity. That is, the drag force of a highly porous sphere can be much less than that for a nonporous sphere. At reduced $h / d, \Omega$ for $\beta>50$ markedly increases. The drag force on nonporous sphere increases rapidly when approaching the plate. As $\beta$ is rather low, the drag force can be little influenced by the existence of the plate. Define the gap $h / d$ at which the drag force starts to be influenced by the impermeable plate as the critical gap $(h / d)_{\mathrm{c}}$. We note that the $(h / d)_{\mathrm{c}}$ decreases with decreasing $\beta$. These findings are consistent with Payatakes and Dassios [23]. 
Fig. 3b depicts the case of $R e=40$, whose basic characteristics are similar to those of $R e=0.1$. However, the corresponding $\Omega$ values are less than those depicted in Fig. 3a, indicating a relatively less significance of the existence of the plate. Second, at evaluated Reynolds number, the $(h / d)_{\mathrm{c}}$ also decreases. Third, at the same $\beta$ value, the $\Omega$ reveals earlier level off as the Reynolds number increases. These are the results of incorporation of an inertial effect that should be put into consideration when the sphere's motion has surpassed the creeping flow limit.

Fig. 3a,b thereby provide information regarding the ratio $\Omega_{0} / \Omega$ versus $h / d$ relationship.

\section{Experimental}

The waste-activated sludge sample was taken from the wastewater treatment plant in Neili Bread Plant, Presidential Enterprise Co., Taoyuan, Taiwan, and was tested within $2 \mathrm{~h}$ of sampling. The chemical oxygen demand (COD), suspended solids (SS) and turbidity data were for the supernatant drawn from the sludge, determined using Environmental Protection Administration (EPA) Taiwan standard methods. The results read $5.6 \mathrm{mg} / \mathrm{l}$ (COD), $7.1 \mathrm{mg} / \mathrm{l}(\mathrm{SS})$ and 1.39 NTU (turbidity). The weight percentage of the sludge sample was $0.7 \%(\mathrm{w} / \mathrm{w})$. True solid density data for sludges were measured by Accupyc Pycnometer 1330 (Micrometritics) as all approximately of $1450 \mathrm{~kg} / \mathrm{m}^{3}$. The floc size ranged from 150 to $10000 \mu \mathrm{m}$, giving a Reynolds number of 0.03-80.

A glass cylinder $(6 \mathrm{~cm}$ diameter and $50 \mathrm{~cm}$ height $)$, sectioned on a side with an attaching plane view glass, was used for floc-settling test. A JVC camera equipped with a close-up lens was used to record the floc motion. A flat plate was installed in the centerline of the settling column. A floc is released carefully from the top of the column. If the floc does not move along the central line, it would be screened out, while only those proceeding along the centerline can pass. The floc diameters normal to and parallel to the vertical direction were measured. The distance between the floc center and flat plate was recorded by analyzing the image. From the distance versus time data, the floc moving speed can be found by numerical differentiation and data smoothing. Fig. 4 depicts schematically the experimental apparatus.

\section{Experimental results}

\subsection{Typical response}

Fig. 5 illustrates the microphotographs of a floc moving toward a flat plate (located at the bottom of Fig. 5). Its terminal velocity in an infinitely large pool $\left(V_{0}\right)$ was measured as $1.16 \mathrm{~mm} / \mathrm{s}$. The floc is apparently not in a perfectly spherical shape. To incorporate the shape effect, the concept of equivalent diameter was adopted, which is defined as the geometric mean of the two principal diameters that measured

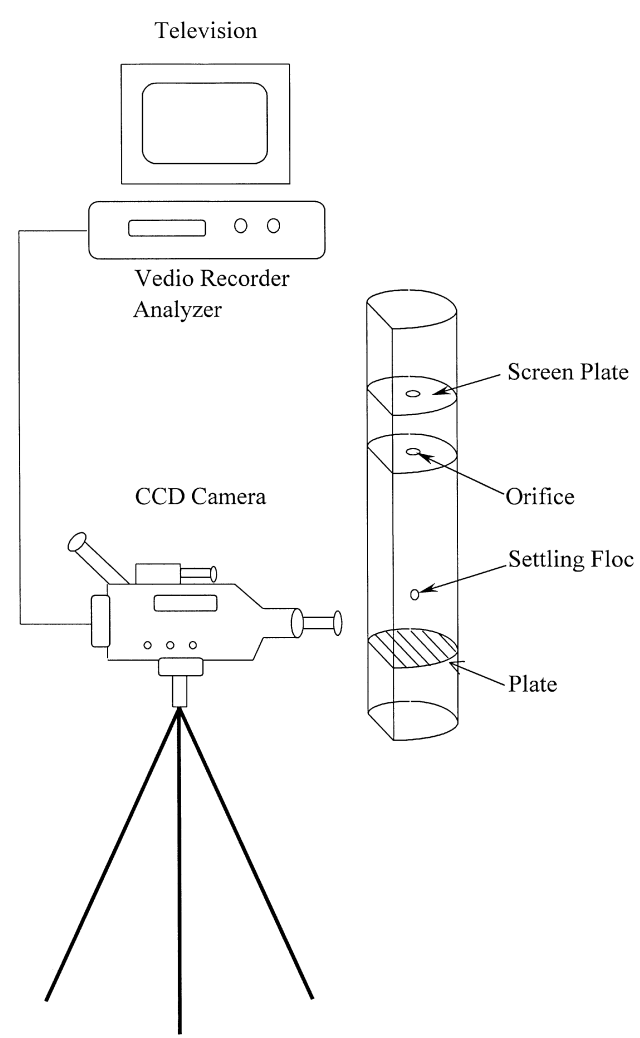

Fig. 4. Schematics of experimental apparatus.

parallel with and normal to the moving direction. The result is $634 \mu \mathrm{m}$. The corresponding Reynolds number is thereby estimated as 0.73 , slightly beyond the creeping-flow regime.

In Fig. 5a, where the floc is $1.47 \mathrm{~mm}(h)$ away from the flat plate, the local moving velocity $(V)$ is $1.159 \mathrm{~mm} / \mathrm{s}$, closely corresponds to the terminal velocity measured in an infinitely large medium. In Fig. 5b, after $0.95 \mathrm{~s}$, the distance among the floc and the plate becomes $0.50 \mathrm{~mm}$, and the floc velocity is reduced to $0.833 \mathrm{~mm} / \mathrm{s}$, signaling the incorporation of plate effects. In Fig. 5c, the floc becomes closer to the plate while the moving speed has been further reduced. Following the movement of the floc, one obtains the $V / V_{0}$ versus $h / d$ relationship. With the help of Eq. (3), Fig. 6a,b depicts some $\Omega_{0} / \Omega$ versus $h / d$ data sets based on their Reynolds number. The solid symbols denote the flocs at $R e<0.1$; while the open symbols, at $R e>1$. The numerical solutions in Fig. 3a,b are also depicted in Fig. 6 for comparison. (Note: the data represented in Fig. 6a,b are the same for the purpose of easy comparison. In addition, since the flocs are very close to the plate are hard to measure accurately, these data are disregarded in the figures.) $\mathrm{Wu}$ et al. [27] estimated the floc permeability according to the analytical solution by Payatakes and Dassios [23], and Burganos et al. [24]. However, the analytical solution cannot be applied to a highly porous object. Furthermore, Wu et al. noted that the analytical solution fails to describe the flocs at a not-too-low Reynolds number. However, in Fig. 6a,b, the fitting between numerical predictions and the experimental 

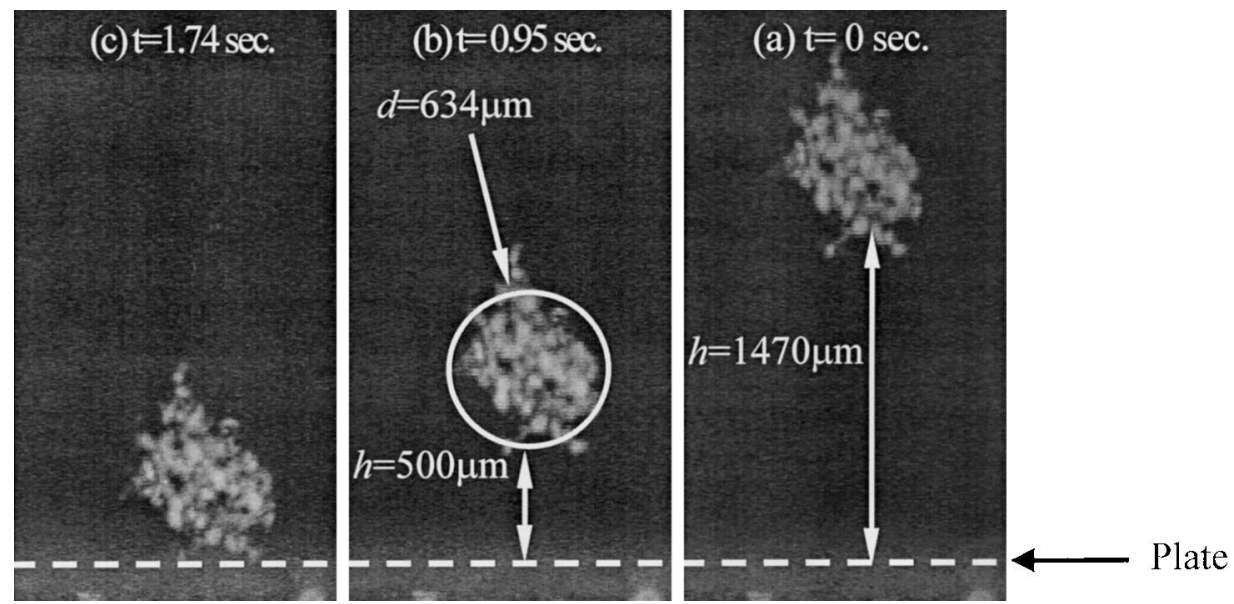

Fig. 5. Floc moving toward a plate. $h$, the distance between the floc and the plate.

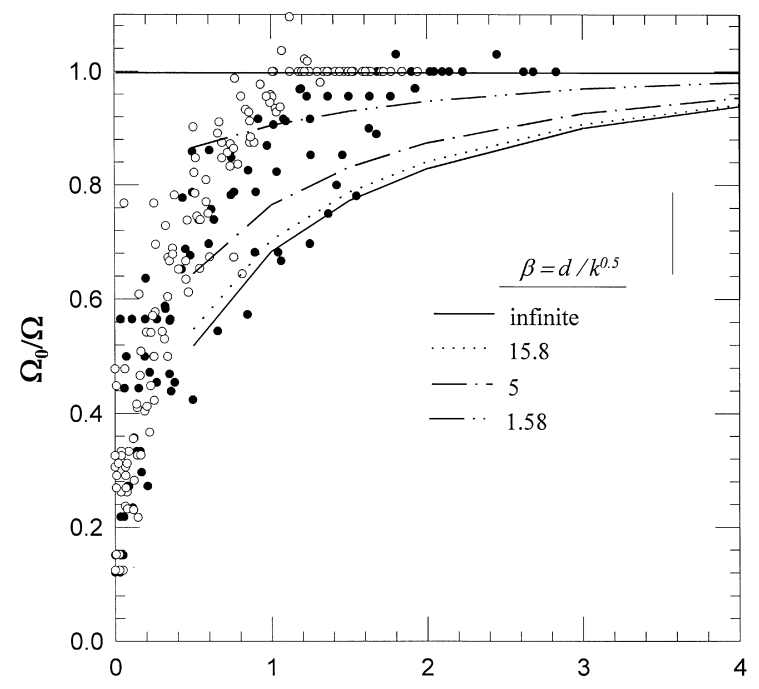

(a)

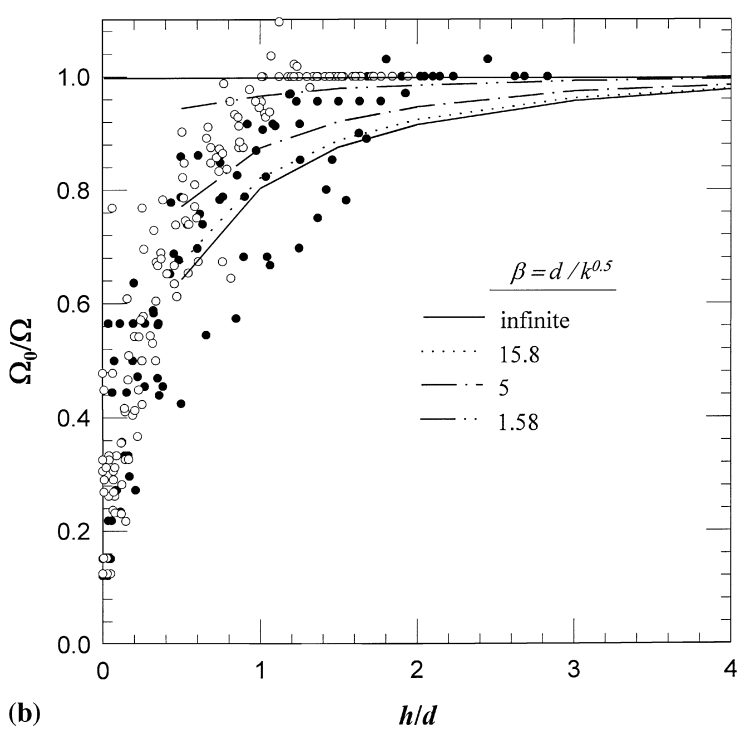

(b)

Fig. 6. $\Omega_{0} / \Omega$ versus $h / d$ plot. Solid symbols, flocs at $R e<0.1$; open symbols, flocs at $R e>1$; Curves in (a) $R e=0.1$; curves in (b) $R e=5$. data can be generally reached. The present numerical solutions thereby more properly interpret the experimental results.

By data fitting, the floc permeability can be estimated. The estimated $\beta$ values read $1.58-5$ for the waste-activated sludge flocs. A weak dependence on floc size is noted. Li and Ganczarczyk [6] defined a critical $\beta$ value of 10.9 , below which the floc can be viewed as a permeable floc. As a result, the present sludges exhibit permeable flocs. From the $\beta$ values and the corresponding floc diameters, the permeability data read $2.5 \times 10^{-9}$ to $9 \times 10^{-6} \mathrm{~m}^{2}$ for floc size ranging from 150 to $10000 \mu \mathrm{m}$.

\section{Floc structure and permeability}

According to the present work, the response of activated sludge flocs that move at low to medium speed correlates with numerical results reported in the preceding sections. A nearly constant $\beta$ value was noted for this sludge over a range of floc sizes. Flocs formed from fine aggregates are generally found to exhibit fractal-like characteristics [28]. Gmachowski $[29,30]$ proposed a model for estimating the $\beta$ parameter by assuming that (a) all flocs in a sludge are fractals exhibiting a unique dimension, and (b) the hydrodynamic drag experienced by a moving floc is attributed mainly to the fluid flow in large pores. The former assumption is possibly valid for some artificial aggregates formed under well-controlled environments, such as those made of polystyrene-latex or gold [31]. Related investigations, however, have confirmed that the flocs generated in natural systems have a multi-level structure [32,33]. The activated sludge floc formation model of Gorczyca and Ganczarczyk [34] consists of the following steps: (1) primary particles form compact flocculi, which are of less porosity and are resistant to shearing; (2) flocculi group together to form microflocs (about $10 \mu \mathrm{m}$ in size), whose porosity is higher than flocculi; and (3) microflocs form weak and highly porous 
flocs (about $100 \mu \mathrm{m}$ in size). Flocculi, microflocs and flocs exhibit different structures, and perhaps different fractal dimensions as well. The simplest picture for a floc can thereby be viewed as a fractal of fractals (but of different dimensions), i.e. a multi-fractal [35]. The structure of flocculi and that of microflocs can be regarded as an internal structure, while that of the entire floc as a global structure. To justify which level of structure affects the permeability is of both academic and practical interest.

Some insights can be obtained from recent works that measure the floc's fractal dimension. Guan et al. [36] employed small-angle laser light-scattering technique for estimating the fractal dimension of activated sludge flocs. For the waste-activated sludge, the fractal dimension obtained (2.23) is markedly greater than that estimated on the basis of the free-settling test (1.4-1.6) [4]. Guan [37] noted that, after vigorous mixing, the fractal dimensions of sludge flocs measured by free-settling tests increase, indicating restructuring action to compact the floc interior. However, the fractal dimensions after shearing determined using light scattering would remain almost unchanged [37]. Such a discrepancy is possibly due to the fact that different measurement techniques actually consider the structure of aggregates of different levels. The light-scattering data in Guan et al. [38] revealed that the characteristic length of the fractal object is approximately $10-20 \mu \mathrm{m}$, correlates well with the size of microflocs rather than the entire floc in the structure model of Gorczyca and Ganczarczyk [34]. Restated, the light scattering data may be reflected of interactions between primary particles in microflocs, rather than by interactions between microflocs in the whole floc.

Pores of all sizes (less than that of the floc size and greater than that of the primary particles) exist in the sludge floc. However, moisture tends to flow through the path of the least resistance [39]. Restated, water would be unlikely to flow into the tiny pores in the microflocs, but rather through the large pores among the microflocs. Permeability measured herein is thereby mainly attributed to the structures of the large pores, or the global structure of the multi-fractal. A nearly constant $\beta$ value obtained herein for flocs of size ranging from 150 to $10000 \mu \mathrm{m}$ indicates that the internal permeability $(k)$ is almost proportional to the square of floc size $\left(d^{2}\right)$. Such an observation further supports the essential role of global structure since the size of the largest pores should be proportional to the floc size $d$.

\section{Conclusions}

This paper provides an estimate of the waste-activated sludge floc interior permeability on the basis of observing the motion of individual floc moving vertically towards an impermeable flat plate. The fluid flow fields around and inside the floc were modeled by the combined Darcy-Brinkman law and the steady-state Navier-Stokes equations. FIDAP numerically solved these equations, from which the hydrodynamic drag was estimated. The experimental data correlate with the numerical solutions regardless of the floc Reynolds number. Floc permeability was then estimated by data fitting. The estimated $\beta$ values read $1.6-5$, indicating that the present flocs exhibit a permeable interior. The structure of waste-activated sludge floc is proposed as a multi-fractal. Permeability measured herein is thereby mainly attributed to the global structure level of the flocs.

\section{Acknowledgements}

The National Science Council, ROC, financially supported this work.

\section{References}

[1] N. Tambo, Y. Watanabe, Water Res. 13 (1979) 409.

[2] T. Mitani, H. Unno, T. Akekata, Jpn. Water Res. 6 (1983) 65.

[3] D.H. Li, J. Ganczarczyk, Water Res. 21 (1987) 257.

[4] D.H. Li, J. Ganczarczyk, Environ. Sci. Technol. 23 (1989) 1385.

[5] D.H. Li, J. Ganczarczyk, Biotechnol. Bioeng. 35 (1990) 57.

[6] D.H. Li, J. Ganczarczyk, Water Environ. Res. 64 (1992) 236.

[7] H. Huang, Clay Clay Miner. 41 (1993) 373.

[8] D.J. Lee, J. Chem. Tech. Biotechnol. 61 (1994) 139.

[9] Q. Jiang, B.E. Logan, Environ. Sci. Technol. 25 (1991) 2031.

[10] D.J. Lee, Journal of the Chinese Institute of Chemical Engineers 25 (1994) 201.

[11] D.J. Lee, Y.H. Hsu, Environ. Sci. Technol. 28 (1994) 1444.

[12] W.T. Hung, I.L. Chang, G.W. Chen, D.J. Lee, Environ. Sci. Technol. 30 (1996) 2391.

[13] D.H. Li, J. Ganczarczyk, Water Res. 22 (1988) 789.

[14] B.E. Logan, J.R. Hunt, Biotechnol. Bioeng. 31 (1988) 92.

[15] D.J. Lee, G.W. Chen, Y.C. Liao, C.C. Hsieh, Water Res. 30 (1996) 541.

[16] K. Matsumoto, A. Suganuma, D. Kunii, Chem. Eng. Sci. 33 (1978) 1554.

[17] S.N. Rogak, R.C. Flagan, J. Colloid Interface Sci. 134 (1990) 206.

[18] S. Chellam, M.R. Wiesner, Water Res. 27 (1993) 1943.

[19] R.M. Wu, W.H. Feng, I.H. Tsai, D.J. Lee, Water Environ. Res. 70 (1998) 1258.

[20] H. Brenner, Chem. Eng. Sci. 16 (1961) 242.

[21] G. Neale, N. Epstein, W. Nader, Chem. Eng. Sci. 28 (1973) 1865.

[22] K. Matsumoto, A. Suganuma, Chem. Eng. Sci. 32 (1977) 445.

[23] A.C. Payatakes, G. Dassios, Chem. Eng. Commun. 58 (1987) 119.

[24] V.N. Burganos, A.C. Michalopoulou, G. Dassios, A.C. Payatakes, Chem. Eng. Commun. 117 (1992) 85.

[25] A.C. Payatakes, H.Y. Park, J. Petrie, Chem. Eng. Sci. 36 (1981) 1319.

[26] S. Veerapaneni, M.R. Wiesner, J. Colloid Interface Sci. 177 (1996) 45.

[27] R.M. Wu, D.J. Lee, Water Res. 32 (1998) 860.

[28] J. Gregory, Filtr. Sep. 35 (4) (1998) 367.

[29] L. Gmachowski, Water Res. 32 (1998) 860.

[30] L. Gmachowski, Water Res. 33 (1999) 1114.

[31] L. Gmachowski, J. Colloid Interface Sci. 178 (1996) 80.

[32] M.M. Clark, J.R.V. Flora, J. Colloid Interface Sci. 147 (1991) 407.

[33] F.D. Sanin, P.A. Vesilind, Water Environ. Res. 68 (1996) 927.

[34] B. Gorczyca, J. Ganczarczyk, personal communication.

[35] D.J. Lee, Water Res. 33 (1999) 1116.

[36] J. Guan, T.D. Waite, R. Wukasch, R. Amal, Proceedings of the IAWQ 19th Biennial International Conference, Vancouver, Canada, vol. 3, 1998, p. 200.

[37] J. Guan, Ph.D. dissertation, University of New South Wales, Australia, 1999.

[38] J. Guan, R. Amal, T.D. Waite, Environ. Sci. Technol. 32 (1998) 3735.

[39] S.C. Chen, J. Chin. Inst. Eng. 21 (1998) 449. 